\title{
Gait-Based Gender Classification Using Persistent Homology
}

\author{
Javier Lamar Leon ${ }^{1}$, Andrea Cerri ${ }^{2, \star}$, Edel Garcia Reyes ${ }^{1}$, \\ and Rocio Gonzalez Diaz ${ }^{3}$
}

1 Patterns Recognition Department, Advanced Technologies Application Center, 7a $\sharp 21812$ e/ 218 y 222, Rpto. Siboney, Playa, C.P. 12200, La Habana, Cuba

$\{$ jlamar, egarcia\}@cenatav.co.cu

2 IMATI - CNR, : via de Marini 6, 16149 Genova, Italy

andrea.cerri@ge.imati.cnr.it

3 Applied Math Dept., School of Computer Engineering, Campus Reina Mercedes, University of Seville, Seville, Spain

rogodi@us.es

\begin{abstract}
In this paper, a topological approach for gait-based gender recognition is presented. First, a stack of human silhouettes, extracted by background subtraction and thresholding, were glued through their gravity centers, forming a 3D digital image $I$. Second, different filters (i.e. particular orders of the simplices) are applied on $\partial K(I)$ (a simplicial complex obtained from $I$ ) which capture relations among the parts of the human body when walking. Finally, a topological signature is extracted from the persistence diagram according to each filter. The measure cosine is used to give a similarity value between topological signatures. The novelty of the paper is a notion of robustness of the provided method (which is also valid for gait recognition). Three experiments are performed using all human-camera view angles provided in CASIA-B database 1 . The first one evaluates the named topological signature obtaining $98.3 \%$ (lateral view) of correct classification rates, for gender identification. The second one shows results for different human-camera distances according to training and test (i.e. training with a human-camera distance and test with a different one). The third one shows that upper body is more discriminative than lower body.
\end{abstract}

Keywords: gait-based recognition, topology, persistent homology, gender classification.

\section{Introduction}

Gender human classification can be obtained based on face [1], voice [2] or gait [3, 4]. Dynamic features when the people walk give the possibility to identify

\footnotetext{
* Supported by the Italian CNR Flagship project INTEROMICS, InterOmics PB05, research unit WP15.

1 http://www.cbsr.ia.ac.cn/english/GaitDatabases.asp1
} 
persons and their gender at a distance, without any interaction from the subjects [5 -7]. This fact can improve the performance of surveillance system intelligent, the analysis of customer information in trade centers, and it can reduce the false positive rate during reidentification of an individual on a wide network cameras. People not only observe the global motion properties while human walk, but they detect motion patterns of local body parts. For instance, females tent to swing their hips more than their shoulders. On the contrary, males tent to swing their shoulders more than their hips [8]. Moreover, males have in general wider shoulders than females [9]. An experiment for human observers to analyze the contributions of different parts of the human body (lower body, upper body and whole body) and to study their discriminative power appears in [3]. According to that experiment, upper body contributes more than lower body to gender classification. In fact, $94.35 \%$ and $67.86 \%$ of correct classification rates, for upper and lower body, respectively, were obtained. In this paper, a modified version of the topological gait signature given in [10, 11] is presented, which is valid for gait and also for gender classification. Besides, an important contribution of the paper are arguments for the robustness of the signature with respect to small input-data perturbations (i.e., perturbations on the stack of silhouettes) are presented. We test this topological signature on the CASIA-B database and compare our method with existing ones for gender recognition.

The rest of the paper is organized as follows. Section 2 is devoted to describe the method for obtaining the topological signature and arguments for its robustness. Experimental results are then reported in Section 3. We conclude this paper and discuss some future work in Section 4 .

\section{Topological Signature for Gender Classification}

In this section, we briefly explain how the topological signature for gait and gender classification is obtained. As we will see below, the filters (ordering of simplices) are given by using functions defined on the simplicial complex $\partial K(I)$ and associated to the given view directions. These functions will be used later for sketching robustness of the topological signature for gait and gender recognition with respect to "small" input-data perturbations. Persistent homology obtained from these filters are represented here in persistence diagram format [12].

\subsection{The Simplicial Complex $\partial K(I)$}

First, the foreground (person) is segmented from the background by applying background modeling and subtraction. The sequence of resulting silhouettes is analyzed to extract one subsequence of representation, which include at least a gait cycle [5].

The $3 \mathrm{D}$ binary digital picture $I=\left(\mathbb{Z}^{3}, B\right)$ (where $B \subset \mathbb{Z}^{3}$ is the foreground), is built by stacking silhouettes of a subsequence of representation, aligned by their gravity centers $(g c)$. See Fig. 1,a and Fig. 1 b. The 3D cubical complex $Q(I)$ associated to $I$ is constructed as follow: Visit all the points $v=(i, j, k) \in B$ from down to up and from left to right. 


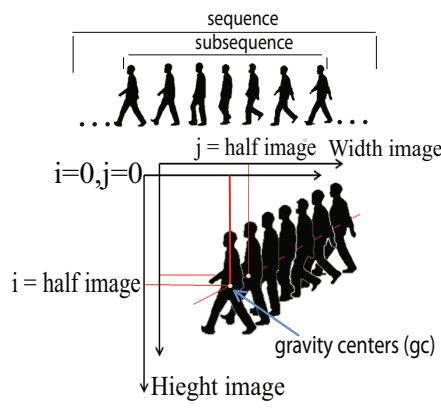

(a)

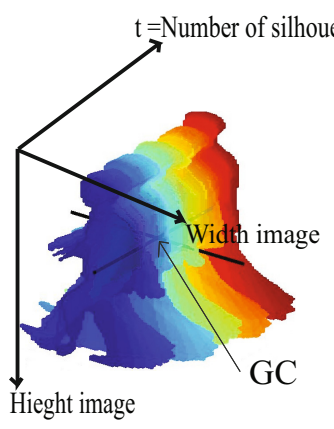

(b)

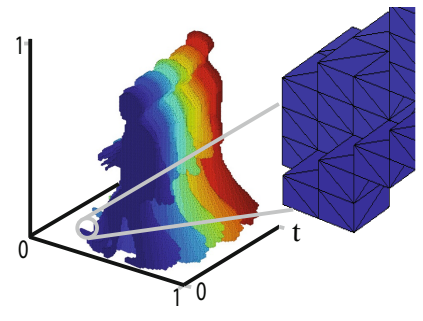

(c)

Fig. 1. (a) Silhouettes aligned by their $g c$. (b) $I=\left(\mathbb{Z}^{3}, B\right)$ obtained from the silhouettes $(G C$ is the gravity center of $I)$. (c) The border simplicial complex $\partial K(I)$.

If the 7 neighbors of $v,\{(i+1, j, k),(i, j+1, k),(i, j, k+1),(i+1, j+1, k)$, $(i+1, j, k+1),(i, j+1, k+1),(i+1, j+1, k+1)\}$, are also in $B$ then, the unit cube formed by these 8 vertices together with all its faces (vertices, edges and squares) are added to $Q(I)$. The simplicial complex $\partial K(I)$ is constructed by selecting all the squares of $Q(I)$ that are faces of exactly one cube in $Q(I)$ and subdividing such squares in two triangles. The faces of each triangle (vertices and edges) are also added to $\partial K(I)$ (see Fig. 1, c). Finally, coordinates of the vertices of $\partial K(I)$ are normalized to coordinates $(x, y, t)$, where $0 \leq x, y \leq 1$ and $t$ is the number of silhouette of the subsequence of representation.

\subsection{Filters for $\partial K(I)$}

The topology of $\partial K(I)$ is, in general, very poor. However, in this subsection we present how, using persistence diagrams, it is possible to get a topological signature from $\partial K(I)$ that captures relations among the parts of the human body when walking, and is robust against small input-data perturbations.

When a view direction $d$ is chosen, two filters for $\partial K(I)$ are obtained as follows. All vertices belonging to $\partial K(I)$ are associated with two filtering functions $f_{+}$and $f_{-}$. For each vertex $v \in \partial K(I), f_{+}(v)$ is the distance between $v$ and the plane normal to $d$ and passing through the origin of the reference frame, while $f_{-}(v)=-f_{+}(v)$. Edges and triangles are associated to the smallest value that $f_{+}\left(\right.$resp. $\left.f_{-}\right)$assumes on their vertices. Being the simplices of $\partial K(I)$ finite in number, we can determine a minimum value for $f_{+}$, say $f_{\min }$, and a maximum one, $f_{\max }$. It is now possible to induce two filters on $\partial K(I)$ by ordering its simplices according to increasing values of $f_{+}$and $f_{-}$, respectively. Denote these filters by $K_{\left[f_{\min }, f_{\max }\right]}=\left\{\sigma_{1}, \ldots, \sigma_{k}\right\}$ and $K_{\left[-f_{\max },-f_{\min }\right]}=\left\{\sigma_{1}^{\prime}, \ldots, \sigma_{k}^{\prime}\right\}$.

\subsection{Persistence Diagrams and Topological Signatures}

Given a simplicial complex $K$, a filtering function $f$, and a filter $\left\{\sigma_{1}, \ldots, \sigma_{k}\right\}$ for $K$, if $\sigma_{i}$ completes a $p$-cycle ( $p$ is the dimension of $\sigma_{i}$ ) when $\sigma_{i}$ is added to 
$K_{i-1}=\left\{\sigma_{1}, \ldots, \sigma_{i-1}\right\}$, then a $p$-homology class $\gamma$ is born at time $i$; otherwise, a $(p-1)$-homology class dies at time $i$. The difference between the birth and death time of a homology class is called its index persistence, which quantifies the significance of a topological attribute. If $\gamma$ never dies, we set its persistence as well as its index persistence to infinity. Drawing a point $(i, j)$ for a homology class that is born at time $i$ and dies at time $j$, we get the $p$-persistence diagram of the filtration, denoted as $\operatorname{Dgm}(f)$. It represents a $p$-homology class by a point whose vertical distance to the diagonal is the persistence. Since always $i<j$, all points lie above the diagonal (see [12]).

In this paper, persistence diagrams are first computed for $K_{\left[f_{\min }, f_{\max }\right]}$ and $K_{\left[-f_{\max },-f_{\min }\right]}$. Then, the diagrams are explored according to a uniform sampling. More precisely, given a positive integer $n$, compute the integer $h=\left\lfloor\frac{k}{n}\right\rfloor$ representing the width of the "window" we use to analyze the persistence diagram. Indeed, for $i=1, \ldots, n$, the $i$-reduced persistence diagram of $K_{\left[f_{\min }, f_{\max }\right]}$ (resp. $K_{\left[-f_{\max },-f_{\min }\right]}$ ) show

(a) Homology classes that are born after $(i-1) \cdot h$ and before $i \cdot h$. Let $\ell$ be the time when such homology class is born. Its reduced life-length is $i \cdot h-\ell$.

Having the reduced persistence diagrams on hand, we can now compute two $n$-dimensional vectors for $K_{\left[f_{\min }, f_{\max }\right]}$ (resp. for $K_{\left[-f_{\max },-f_{\min }\right]}$ ) whose $i$-entry corresponds to:

1. the sum of the reduced life-lengths for the 0 -homology classes $s u m H_{0}$

2. the sum of the reduced life-lengths for the 1-homology classes $s u m H_{1}$.

These two collections of two $n$-dimensional vectors, represent the topological signature for a gait subsequence associated with a fixed view direction.

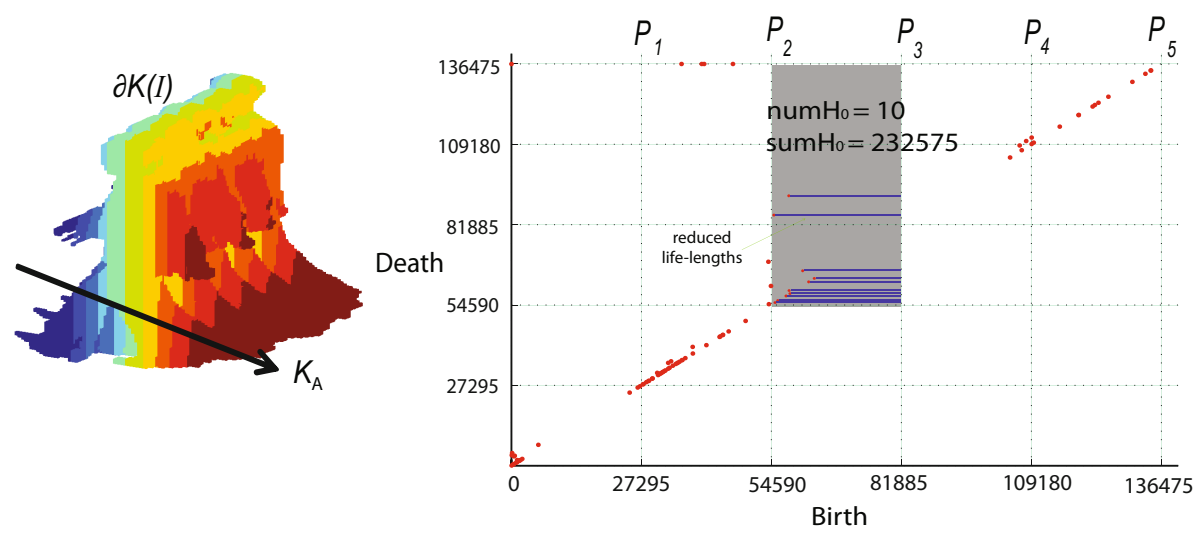

Fig. 2. An example of computation of the first element of a topological signature

For example, consider $K_{A}$ given in Fig. 2 which consists in 136475 triangles. We perform $n=5$ uniform cuts on the 0 -persistence diagram. The sum of the 
reduced life-lengths for the 0 - homology classes $\left(\right.$ num $\left._{0}=10\right)$ that were born in time $54560 \leq t<81885$ are sum $H_{0}=232575$ (blue lines in Fig. 2). The first element of the topological signature: $V_{1}$ is, $\{473625,813786,232575,10039,203958\}$.

\subsection{Comparing Topological Signatures}

The topological signatures for two gait subsequences associated with a fixed view direction, say $V=\left\{V_{1}, \ldots, V_{4}\right\}$ and $W=\left\{W_{1}, \ldots, W_{4}\right\}$, can be compared according to the following procedure: for every $i=\{1, \ldots, 4\}$ compute:

$$
S_{i}=\frac{V_{i} \cdot W_{i}}{\left\|V_{i}\right\| \cdot\left\|W_{i}\right\|} .
$$

which is the cosine of the angle between the vectors $V_{i}$ and $W_{i}$. Observe that $0 \leq S_{i} \leq 1$ since the entries of both vectors are always non-negative. Then, the total similarity value for two gait subsequences, $\mathrm{O}_{1}$ and $\mathrm{O}_{2}$, considering a fixed view direction, is the sum of the 4 similarity measures computed before:

$$
S\left(O_{1}, O_{2}\right)=S_{1}+S_{2}+S_{3}+S_{4}
$$

\subsection{Robustness}

In this subsection, we briefly sketch a notion of robustness for our topological signature with respect to small input-data perturbations. Fix a view direction $d$ and an associated filtering function $f$. The assumption here is that the inputdata perturbations can be modeled as perturbations of the function $f$. We could think, for example, of small perturbations in fixing $d$, as well as noise in the computation of $f$. More precisely, consider two functions $f, g: \partial K(I) \rightarrow \mathbb{R}$ such that

$$
\max _{\sigma \in \partial K(I)}|f(\sigma)-g(\sigma)| \leq \varepsilon
$$

with $\varepsilon$ being a small positive real number. Let $K_{f}=\left\{\sigma_{1}, \ldots, \sigma_{k}\right\}$ and $K_{g}=$ $\left\{\sigma_{1}^{\prime}, \ldots, \sigma_{k}^{\prime}\right\}$ be the filters associated with the increasing values of $f$ and $g$, respectively 2 . Assume also that all homology classes fulfill either condition $(a)$ : Such an assumption is actually mild, and can be achieved quite easily in practice (e.g., by slightly perturbing the values of $f$ ). Then, the stability of persistence diagrams [13] implies that the birth- and the death-times, with respect to $g$, of each homology class, cannot differ more than $\varepsilon$ from those with respect to $f$. Therefore, if $\varepsilon$ is sufficiently small, it follows that

- If a homology class fulfills condition $(a)$ for $K_{f}$, the same occurs for $K_{g}$.

Moreover, the same stability result in [13] implies that new homology classes, living no longer than $2 \varepsilon$, could appear, as well as old classes living shorter than $2 \varepsilon$ may vanish. These events could sensibly change the number of homology classes satisfying condition $(a)$. Nevertheless, considering such classes according to their reduced life-length, as specified above, guarantees the robustness of our topological signatures.

\footnotetext{
${ }^{2}$ Similar arguments hold if considering filters associated with the decreasing values of $f$ and $g$.
} 


\section{Experimental Results}

In this section, we show the performance of the proposed method on gait sequences from the CASIA-B database, which contains 124 subjects, 91 males and 31 females. There are 6 walking sequences for each person. CASIA-B database provides image sequences with background subtraction for each person.

To avoid bias, 31 males were randomly selected. The 62 subjects were then divided in 31 disjoint sets, each containing 2 subjects (a male and a female). Only one of these 31 sets was sued to test. The remaining 30 sets were used for training. The correct classification rate (CCR) is the average of the 31 possible combinations.

The experimental protocol was made according to [3, 4]. In this experiment, a subsequence of representation corresponds to the whole sequence, which has two gait cycle as average. We have fixed $n=24$ and used 3 view directions. The first one is vertical (i.e. parallel to axis $y$ ). The second one forms 45 degrees with axes $x$ and $y$ and 90 degrees with axis $t$. The third one is parallel to axis $t$. See Fig. 3. In each experiment, the results of our method are compared with the methods presented in [3, 4].

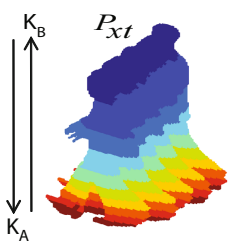

(a)

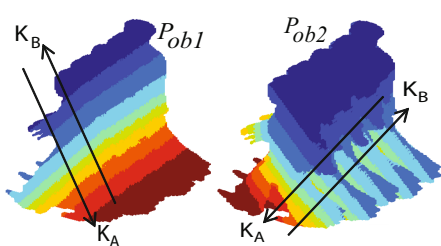

(c)

Fig. 3. View directions used in the experiments

\subsection{Experiment 1}

The aim of this experiment is to evaluate the topological signature for gender classification. Table 1 shows the 31 -fold-cross-validation of CCR for the whole body using the 11 view directions provided by the CASIA-B database. The first line of the table refers to the camera view angle. This way, 0 degrees means that the person is in front to the camera and walking to the camera, 90 degrees means that the person is walking lateral to the camera (lateral view), and 180 degrees means that the person is back to the camera and walking away the camera. We can see that the topological signature provides better results for the lateral view. This agrees with [7].

\subsection{Experiment 2}

In this experiment we show that our topological signature is robust with respect to scaling. Images form CASIA-B database of size $320 \times 240$ are scaled to 
Table 1. Correct classification rates (CCR in \%) for the whole body

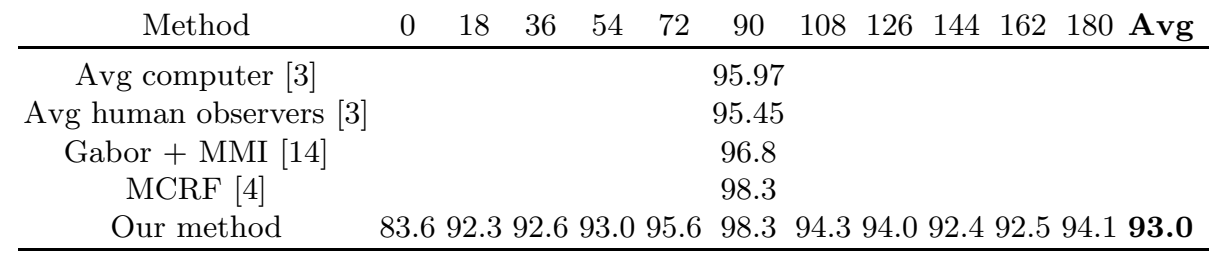

$160 \times 120$. Table 2 shows results considering different scales for training and test. For example, if images of size $320 \times 240$ are used for training and images of size $160 \times 120$ are using for test, then we obtain $98.0 \%$ of CCR. Nevertheless, if images of size $160 \times 120$ are used for training and images of size $320 \times 240$ are using for test, then we obtain $95.6 \%$ of CCR.

Table 2. Correct classification rates (CCR in \%) using different sizes of the images for training and test

\begin{tabular}{c|ccc} 
& \multicolumn{3}{|c}{ Test } \\
\hline \multirow{3}{*}{ Training } & $320 \times 240$ images & $160 \times 120$ images \\
& $160 \times 120$ images & 98.3 & 98.00 \\
images & 95.6 & 97.5
\end{tabular}

\subsection{Experiment 3}

The aim of this experiment is to compare gender classifications using only upper or lower body. According to Table 3, our results confirm that upper body contributes more than lower body to gender classification for both original (320x240) and scaled $(160 x 120)$ images. This agrees with the results obtained by human observers in [3].

Table 3. Correct classification rates (CCR in \%) for lower and upper body from lateral view for original $(320 \times 240)$ and scaled $(160 \times 120)$ images

\begin{tabular}{lc}
\hline Method & CCR (lateral view) \\
\hline Human observers (lower body) $(320 \times 240$ images $)[3]$ & 67.86 \\
Our method (lower body) $(320 \times 240$ images $)$ & 88.1 \\
Our method (lower body) $(160 \times 120$ images $)$ & 87.0 \\
& \\
Human observers (upper body $(320 \times 240$ images $)[3]$ & 94.35 \\
Our method (upper body) $(320 \times 240$ images $)$ & 96.0 \\
Our method (upper body) $(160 \times 120$ images $)$ & 95.5 \\
\hline
\end{tabular}




\section{Conclusion and Future Work}

In this paper, a representation based on topological invariants, previously used for gait based human identification at a distance, is used for a gender classification task. Arguments for the robustness of the method with respect to small input-data perturbations are given. It should be noticed that the view direction should be selected according to the camera view angle to improve the results. The method has been implemented in $\mathrm{C}++$ and has been tested in real-time real-life scenery in [11]. Our future work consists in trying to improve our results for camera view angles different to lateral view selecting the appropriate view direction, and to adapt our method to occlusions.

\section{References}

1. Golomb, B.A., Lawrence, D.T., Sejnowksi, T.J.: SEXNET: A neural network identifies sex from human faces. In: Lippmann, R.P., Moody, J.E., Touretzky, D.S. (eds.) Advances in Neural Information Processing Systems, vol. 3, pp. 572-579. Morgan Kaufmann Publishers, Inc. (1991)

2. Harb, H., Chen, L.: Gender identification using a general audio classifier. In: Proceedings of the 2003 International Conference on Multimedia and Expo, ICME 2003, vol. 1, pp. 733-736. IEEE (2003)

3. Yu, S., Tan, T., Huang, K., Jia, K., Wu, X.: A study on gait-based gender classification. IEEE Trans. Image Processing 18(8), 1905-1910 (2009)

4. Hu, M., Wang, Y., Zhang, Z., Zhang, D.: Gait-based gender classification using mixed conditional random field. IEEE Transactions on Systems, Man, and Cybernetics, Part B 41(5), 1429-1439 (2011)

5. Nixon, M.S., Carter, J.N.: Automatic recognition by gait. Proc. of IEEE 94(11), 2013-2024 (2006)

6. Kale, A., Sundaresan, A., Rajagopalan, A.N., Cuntoor, N.P., Chowdhury, A.K.R., Kruger, V., Chellappa, R.: Identification of humans using gait. IEEE Trans. Image Processing 13(9), 1163-1173 (2004)

7. Goffredo, M., Carter, J., Nixon, M.: Front-view gait recognition. In: Biometrics: Theory, Applications and Systems, September 29-October 1, pp. 1-6 (2008)

8. Aggarwal, J.K., Cai, Q.: Human motion analysis: A review. Computer Vision and Image Understanding 73(3), 428-440 (1999)

9. Mather, G., Murdoch, L.: Gender discrimination in biological motion displays based on dynamic cues. Proceedings of Biological Sciences 258(1353) (1994)

10. Lamar-León, J., García-Reyes, E.B., Gonzalez-Diaz, R.: Human gait identification using persistent homology. In: Alvarez, L., Mejail, M., Gomez, L., Jacobo, J. (eds.) CIARP 2012. LNCS, vol. 7441, pp. 244-251. Springer, Heidelberg (2012)

11. Lamar, J., Garcia, E., Gonzalez-Diaz, R., Alonso, R.: An application for gait recognition using persistent homology. Electronic Journal Image-A 3(5) (2013)

12. Edelsbrunner, H., Harer, J.: Computational Topology - an Introduction. American Mathematical Society (2010)

13. Cohen-Steiner, D., Edelsbrunner, H., Harer, J.: Stability of persistence diagrams. Discrete \& Computational Geometry 37(1), 103-120 (2007)

14. Hu, M., Wang, Y., Zhang, Z., Wang, Y.: Combining spatial and temporal information for gait based gender classification. In: 20th International Conference on Pattern Recognition (ICPR), pp. 3679-3682. IEEE (2010) 